
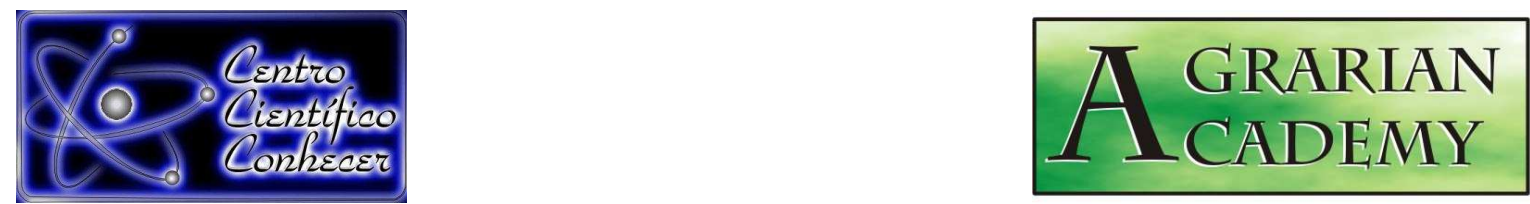

\title{
CULTIVO DA TECA: CARACTERÍSTICAS DA ESPÉCIE PARA IMPLANTAÇÃO E CONDUÇÃO DE POVOAMENTOS FLORESTAIS
}

\footnotetext{
Allan Libanio Pelissari ${ }^{1}$, Pompeu Paes Guimarães ${ }^{2}$, Alexandre Behling ${ }^{3}$, Ângelo Augusto Ebling ${ }^{4}$

${ }^{1}$ Doutorando, Programa de Pós-graduação em Engenharia Florestal da Universidade Federal do Paraná, Curitiba, Paraná, Brasil e-mail: allanpelissari@gmail.com

${ }^{2}$ Professor da Universidade Federal Rural do Semi-Árido, Mossoró, Rio Grande do Norte, Brasil

${ }^{3}$ Doutorando, Programa de Pós-graduação em Engenharia Florestal da Universidade Federal do Paraná, Curitiba, Paraná, Brasil

${ }^{4}$ Doutorando, Programa de Pós-graduação em Engenharia Florestal da Universidade Federal do Paraná, Curitiba, Paraná, Brasil

Contato: pompeupaes@yahoo.com.br (avaliador)
}

Recebido em: 03/01/2014 - Aprovado em: 04/04/2014 - Publicado em: 12/04/2014

\section{RESUMO}

Nas últimas décadas, os plantios de teca têm alcançado destaque no setor florestal brasileiro, contudo, o manejo da espécie é, ainda, por vezes baseado em conhecimentos insuficientes e inadequados, visto que apresenta, em geral, crescimento superior no continente americano, o que dificulta estabelecer comparações entre os regimes de manejo praticados no mundo e os resultados das práticas silviculturais em locais com características edafoclimáticas distintas. Com isso, o objetivo do presente trabalho foi reunir as informações contidas nas principais pesquisas científicas conduzidas em diferentes localidades no mundo aptas ao cultivo da teca, visando, dessa forma, padronizar o conhecimento silvicultural e de manejo da espécie, bem como gerar perspectivas futuras para a condução de maciços florestais no Brasil. Observa-se, atualmente, no mercado nacional e internacional, que as plantações de teca são consideradas alternativas rentáveis e sustentáveis à exploração de espécies nativas para a obtenção de madeira de uso nobre e para diversos outros fins, tornandoa destaque na balança comercial do setor florestal brasileiro e uma oportunidade na conquista de mercados exigentes por produtos florestais de qualidade.

PALAVRAS-CHAVE: manejo florestal, madeira de alto valor econômico, práticas silviculturais, Tectona grandis

\section{TEAK CULTIVATION: SPECIE CHARACTERISTICS FOR THE FORMATION AND CONDUCTION OF FOREST STANDS}

\section{ABSTRACT}

In recent decades, the teak stands have attained featured in the Brazilian forest sector, 
however, the management of the species is still based in insufficient and inadequate knowledge, because the teak has, in general, higher growth in the American continent, which complicates comparisons between management regimes practiced around the world and the results of silvicultural practices in locations with different climate and soil characteristics. Thus, the aim of this study was to gather the information contained in the major scientific research conducted in different locations around the world for the teak cultivation, aiming at standardize the management and silvicultural knowledge of the species, as well as generate future perspectives for conducting forest stands in Brazil. Currently, it is observed in domestic and international market which teak plantations are considered profitable and sustainable alternatives to the exploitation of native species, and for obtaining wood of noble use and many other purposes, making them highlighted in the trade balance of the Brazilian forest sector and an opportunity in the conquest of demanding markets for forest products quality.

KEYWORDS: Tectona grandis, forest management, silvicultural practices, timber high economic value.

\section{INTRODUÇÃO}

A teca é uma espécie natural do continente asiático e cultivada em diversas regiões da África e das Américas. Na atualidade, é considerada uma alternativa às espécies de elevado valor econômico para o suprimento sustentável das indústrias de base florestal, cujo principal produto é a madeira de qualidade, utilizada em móveis de luxo e na construção naval, além disso, há a possibilidade de comercialização da produção desde os primeiros desbastes, a qual é voltada, principalmente, à energia.

Essa versatilidade quanto ao uso, torna a teca uma importante fonte de renda e um investimento lucrativo aos seus produtores (ÂNGELO et al., 2009; NEWBY et al., 2012), correspondendo, atualmente, a aproximadamente 68 mil hectares de plantios no Brasil, com crescimento anual de $1 \%$ em área plantada, principalmente nos estado de Mato Grosso e Pará (ABRAF, 2012).

Nas últimas décadas, os plantios de teca têm alcançado destaque no setor florestal brasileiro, entretanto, o manejo da espécie é, por vezes, baseado em conhecimentos insuficientes e inadequados, visto que apresenta, em geral, crescimento superior no continente americano, o que dificulta estabelecer comparações entre os regimes de manejo praticados no mundo e as respostas das práticas silviculturais em locais com características edafoclimáticas distintas.

Com isso, o objetivo do presente trabalho foi reunir as informações contidas nas principais pesquisas científicas conduzidas em diferentes localidades no mundo aptas ao cultivo da teca, visando, dessa forma, padronizar o conhecimento silvicultural e de manejo da espécie, bem como gerar perspectivas futuras para a condução de maciços florestais no Brasil.

\section{CARACTERÍSTICAS BOTÂNICAS DA ESPÉCIE}

O gênero Tectona, pertencente à família Lamiaceae, antigamente Verbenaceae, é composto pelas espécies Tectona grandis L. f.; Tectona hamiltoniana Wall.; Tectona philippinensis Benth. \& Hook.; Tectona ternifolia Buch.-Ham. ex Wall.; e Tectona theka Lour (TROPICOS, 2013). Dentre essas, somente a Tectona grandis, popularmente conhecida como teca, teak, teck, ojati, may sak ou tiek (FIGUEIREDO et 
al., 2005a), alcançou destaque mundial, devido a madeira de qualidade voltada, principalmente, para usos nobres (CRUZ, 2005; COSTA et al., 2007).

Em ambientes naturais, a teca apresenta tronco retilíneo (Figura 1), com casca áspera e fina, de aproximadamente $1,2 \mathrm{~cm}$, que se desprende em placas (CHAVES \& FONSECA, 1991). É uma espécie com grau alto de deciduidade foliar, com folhas opostas, elípticas, coriáceas, pecíolos curtos ou ausentes, e ápices e bases agudas (Figura 1). Nos indivíduos adultos, as folhas possuem, em média, de 30 a $40 \mathrm{~cm}$ de comprimento por $25 \mathrm{~cm}$ de largura, porém, nos indivíduos mais jovens, com até três anos de idade, as folhas podem atingir o dobro dessas dimensões (FIGUEIREDO et al., 2005a; COSTA et al., 2007).

A inflorescência da teca é composta por cachos, na forma de panículas, com 700 a 3.500 flores brancas e pequenas (Figura 1). Desse total, apenas $1 \%$ a $2 \%$ se desenvolvem em frutos, os quais são constituídos por uma membrana fina que reveste uma estrutura esférica de 5 a $20 \mathrm{~mm}$ de diâmetro. $O$ fruto da teca é do tipo drupa subglobosa e tetralocular, contendo de uma até, mais raramente, quatro sementes por lóculo. Essas sementes são pequenas, delicadas e oleaginosas, com 5 a $6 \mathrm{~mm}$ de comprimento (CALDEIRA et al., 2000; BEZERRA, 2009).

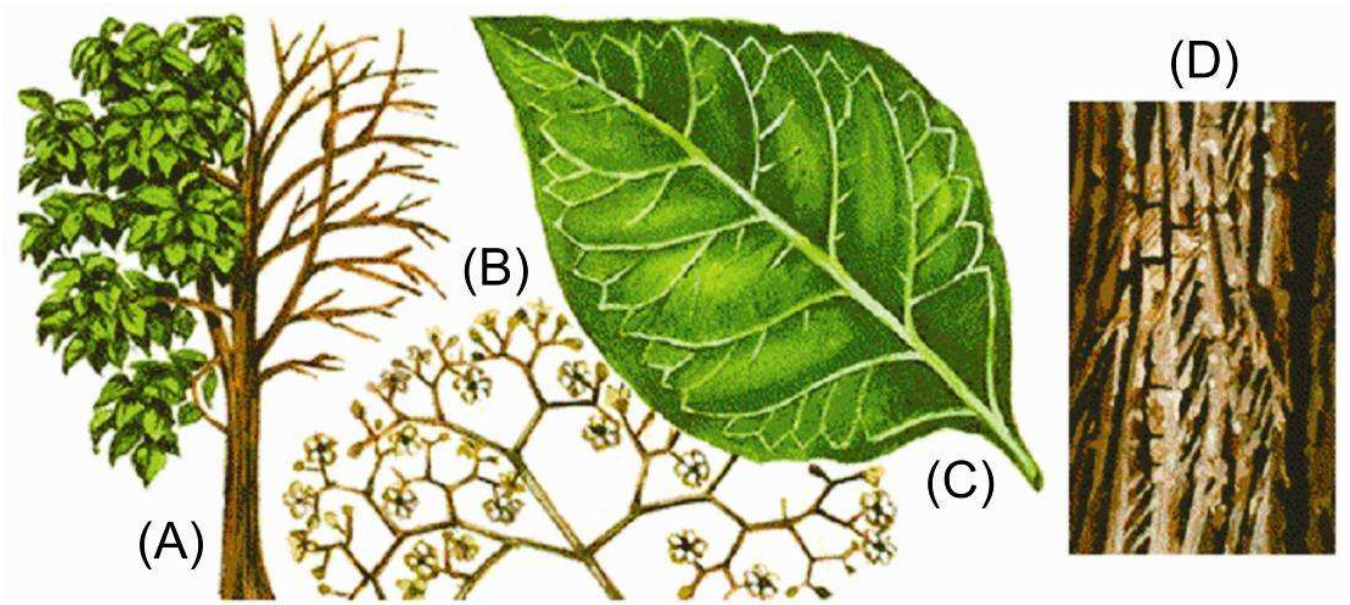

FIGURA 1 - Representação das estruturas morfológicas da teca: tronco e galhos $(A)$, inflorescência $(B)$, folha $(C)$ e casca (D).

Fonte: adaptado de CALDEIRA (2004).

\section{DISTRIBUIÇÃO GEOGRÁFICA MUNDIAL}

A teca é uma espécie do sul e sudeste do continente asiático, com distribuição natural descontinua na Índia, Mianmar, Tailândia e Laos, entre os paralelos $9^{\circ}$ e $25^{\circ}$ de latitude norte (Figura 2), e introduzida, há centenas de anos, na Indonésia e Sri Lanka. Atualmente, apresenta uma distribuição relativamente ampla, sendo cultivada em diversas regiões da África e das Américas do Sul e Central, ocupando espaço de destaque no mercado entre as principais espécies produtoras de madeira tropical (CATIE, 1986; TANAKA et al., 1998; KRISHNAPILLAY, 2000; PANDEY \& BROWN, 2000; BERMEJO, et al. 2004; RUGMINI \& JAYARAMAN, 2009; NOCETTI et al., 2011). 


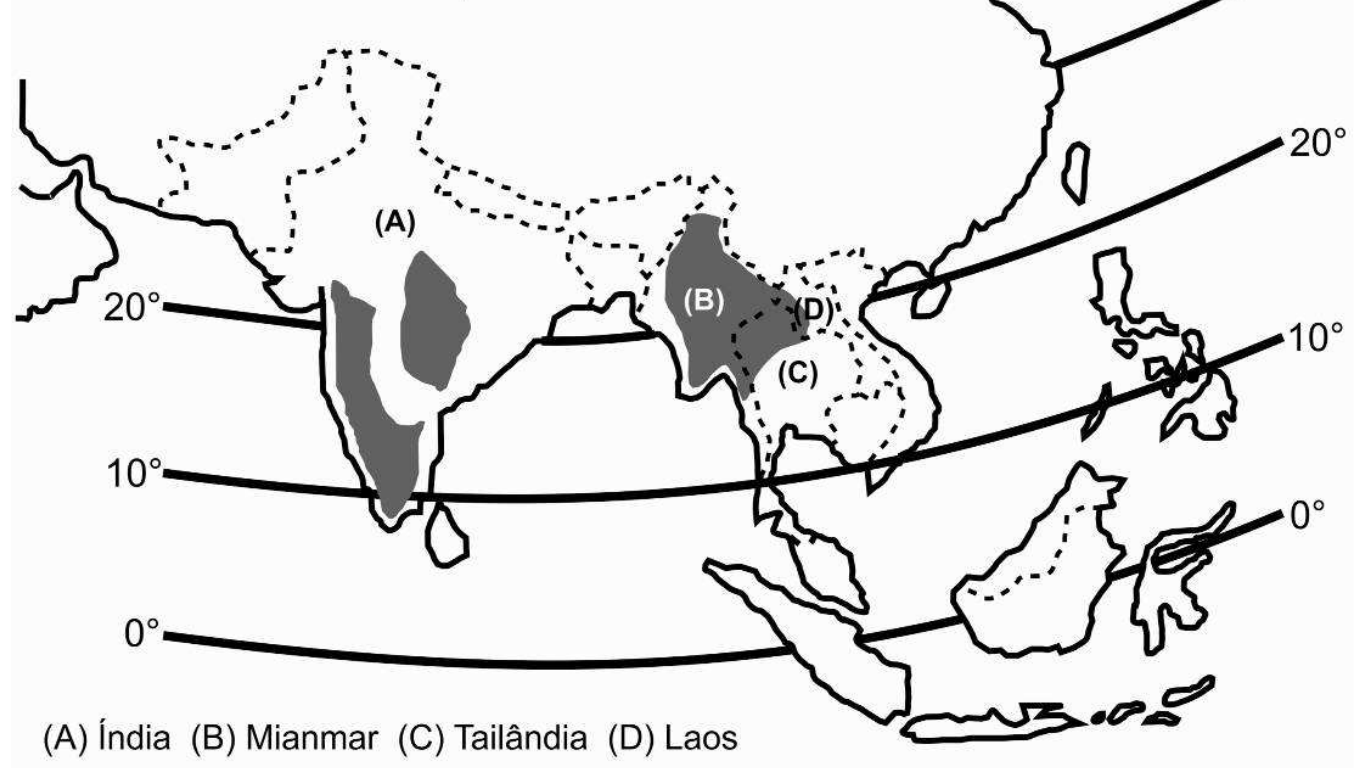

FIGURA 2 - Distribuição natural da teca no continente asiático.

Fonte: adaptado de KAOSA-ARD (1981) e TANAKA et al. (1998).

As florestas naturais de teca representam uma área relativamente limitada e de participação baixa na produção de madeira (KRISHNAPILLAY, 2000; MITTELMAN, 2000; NAIR \& SOUVANNAVONG, 2000). Entretanto, somente a partir da proibição da exploração das florestas nativas dos principais fornecedores, como a Índia em 1986 e Laos e Tailândia em 1989, os povoamentos artificiais de teca alcançaram destaque como importante fonte de madeira e com potencial para suprir a demanda mundial (KRISHNAPILLAY, 2000; MITTELMAN, 2000; PANDEY \& BROWN, 2000), principalmente nos trópicos, devido ao maior potencial de crescimento e produtividade (VAIDES et al., 2005).

Estima-se que, aproximadamente, há 4,3 milhões de hectares cultivados com teca, sendo $83 \%$ concentrados na Ásia, $11 \%$ na África e $6 \%$ na América tropical (CAMINO \& MORALES, 2013). A Índia e a Indonésia destacam-se, respectivamente, com $44 \%$ e $31 \%$ da área plantada com a espécie no continente asiático (SHUKLA et al., 2011), enquanto Mianmar é o único país que ainda depende de florestas naturais para a produção de madeira de teca (NAIR \& SOUVANNAVONG, 2000), ao passo que no Brasil, o interesse na espécie, como alternativa aos plantios florestais tradicionais (Figura 3), vem crescendo muito atualmente nas regiões Centro-Oeste e Norte do país (FIGUEIREDO et al., 2005b; LIMA et al., 2009; SCHUHLI \& PALUDZYSZYN FILHO, 2010), principalmente no estado de Mato Grosso que, em 2007, já apresentava 48.562 ha cultivados com a espécie (SHIMIZU et al., 2007). 

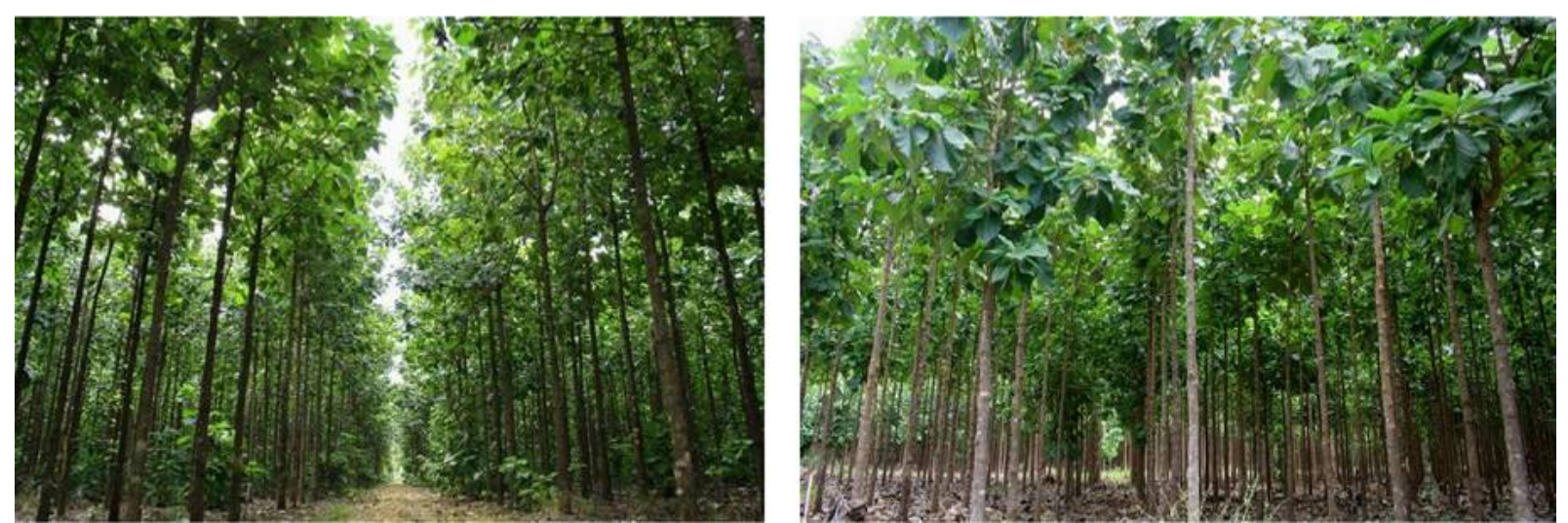

FIGURA 3 - Plantios de teca no estado de Mato Grosso, Brasil.

Fonte: TECA DO BRASIL (2013).

\section{CONDICIONANTES EDAFOCLIMÁTICAS}

A área de ocorrência natural da teca restringe-se a regiões com clima de monção (LAMPRECHT, 1990). Entretanto, o seu crescimento varia de acordo com as condições edáficas e climáticas locais, principalmente a precipitação, umidade relativa do ar e temperatura (SINHA et al., 2011). A teca apresenta maior taxa de crescimento em localidades com precipitação anual de $1.250 \mathrm{~mm}$ a $3.750 \mathrm{~mm}$, associado a um período de três a cinco meses de seca, temperaturas mínimas de $13{ }^{\circ} \mathrm{C}$ a $17 \stackrel{\circ}{\circ} \mathrm{C}$ e máximas de $39^{\circ} \mathrm{C}$ a $43^{\circ} \mathrm{C}$ e altitudes desde o nível do mar até cerca de $1000 \mathrm{~m}$ (CATIE, 1986; CHAVES \& FONSECA, 1991; FLOORS, 1997; KAOSA-ARD, 1998; PANDEY \& BROWN, 2000). É uma espécie exigente em luz e sensível a geadas (SALAZAR \& ALBERTIN, 1974; KAOSA-ARD, 1998; CATIE, 1986; UPADHYAY et al., 2005) e, também, aos ventos fortes (VÁSQUEZ \& UGALDE, 1995; ALVARADO, 2006).

$\mathrm{Na}$ América Central, o maior crescimento da teca é observado nos sítios com altitudes inferiores a $500 \mathrm{~m}$, com uma estação seca de quatro a seis meses ao ano, temperatura média entre $23{ }^{\circ} \mathrm{C}$ e $27{ }^{\circ} \mathrm{C}$ e precipitação média anual de $1.300 \mathrm{~mm}$ a 2.500 mm (CATIE, 1986; GONZÁLEZ, 2004). No Brasil, a espécie é cultivada em locais com precipitação média anual entre $1.500 \mathrm{~mm}$ a $2.750 \mathrm{~mm}$, temperaturas máximas de $35 \stackrel{\circ}{\circ}$ a $40 \stackrel{\circ}{\circ}$ e mínimas de $15 \stackrel{\circ}{ } \mathrm{C}$ a $20 \stackrel{\circ}{\circ}$, com três a quatro meses de período seco, o que são consideradas condições ideais ao seu desenvolvimento da espécie (OLIVEIRA, 2003; BEHLING, 2009).

A teca se desenvolve em uma grande variedade de solos e formações geológicas (TONINI et al., 2009), porém tem preferência pelos de textura francoarenosos a argilosos (CHAVES \& FONSECA, 1991; OMBINA, 2008), profundos e de boa drenagem, com terrenos planos, ou pouco declivosos, e férteis (VÁSQUEZ \& UGALDE, 1995; CENTENO, 1997; KAOSA-ARD, 1998; TANAKA et al., 1998; MONTERO et al., 2001).

$\mathrm{O} \mathrm{pH}$ é uma das mais importantes propriedades do solo para a teca $(\mathrm{ZECH}$ \& DRECHSEL, 1991; PELISSARI et al., 2012), estando diretamente ligado à disponibilidade de nutrientes do solo (LIMA et al., 2010). Entretanto, não há consenso entre os seus níveis ideais para o desenvolvimento da teca. Autores como KAOSA-ARD (1998) e TANAKA et al. (1998) afirmaram que a espécie tem preferência por pH ligeiramente ácido à alcalino, de 6,5 a 7,5. Enquanto OMBINA (2008) determinou que o 
intervalo de $\mathrm{pH}$ de 6,0 a 8,0 é considerado como o melhor para os povoamentos da teca na Índia e Mianmar, a passo que MOLLINEDO GARCIA (2003) e GONZÁLEZ (2010) estabeleceram que a teca não deva ser cultivada em sítios com pH inferior a 5,5, pois o seu crescimento é limitado pela redução na disponibilidade de diversos elementos essenciais no solo.

A teca é eficiente na utilização do fósforo (MATA, 1999), com alto poder de assimilação (VALLEJOS BARRA, 1996) para o desenvolvimento do seu sistema radicular (BEHLING, 2009). Além deste elemento, a disponibilidade do potássio tende a influenciar o crescimento da espécie (CASTELLANOS, 2006) devido a sua relação com os processos metabólicos das plantas (MORAES et al., 2008). Segundo MOLLINEDO GARCIA (2003), níveis de potássio e fósforo, inferiores a aproximadamente $4,5 \mathrm{mg} \cdot \mathrm{dm}^{-3}$ e $0,5 \mathrm{mg} \cdot \mathrm{dm}^{-3}$, respectivamente, são críticos ao desenvolvimento da teca, sendo necessário incorporá-los ao solo com a aplicação de fertilizantes.

Além disso, é considerada uma espécie altamente exigente em cálcio (TANAKA et al., 1998; GONZÁLEZ, 2004; PELISSARI et al., 2012), respondendo significativamente ao acréscimo deste elemento no solo (MATRICARDI, 1989). Em geral, os melhores sítios para a teca estão associados a um conteúdo de cálcio no solo superior a $10 \mathrm{cmol}_{\mathrm{c}} \cdot \mathrm{dm}^{-3}$ nos primeiros horizontes (VÁSQUEZ \& UGALDE, 1995; MOLLINEDO GARCIA, 2003). Enquanto concentrações baixas de magnésio no solo são suficientes para atender as exigências da teca (MATRICARDI, 1989), em que sítios com teores de magnésio inferiores a $5 \mathrm{cmol}_{\mathrm{c}} \cdot \mathrm{dm}^{-3}$ podem limitar o crescimento da teca (MOLLINEDO GARCIA, 2003).

A espécie é sensível à acidez no solo sob a forma de elevadas concentrações de alumínio trocável (MATRICARDI, 1989; MOLLINEDO GARCIA, 2003; PELISSARI et al., 2012). Isso pode causar deformidade na divisão celular, diminuição da respiração das raízes, interferência na captação e transporte de nutrientes (OMBINA, 2008) e taxa baixa de sobrevivência das plantas (SILVA et al., 2011). VAIDES LÓPEZ (2004) destacou que a teca apresenta produtividade baixa em sítios com teores superiores a $1,3 \mathrm{cmol}_{\mathrm{C}} \cdot \mathrm{dm}^{-3}$.

A matéria orgânica no solo possui um papel importante para o manejo do solo em plantios de teca, devido ao fornecimento de nutrientes e a manutenção do $\mathrm{pH}$ do solo (SUZUKI et al., 2007). A sua presença em teores elevados promove respostas significativas ao crescimento da teca, sendo mais eficiente nas camadas superficiais do solo, onde grande parte do sistema radicular da teca está presente (MATRICARDI, 1989).

\section{PRODUTOS E USOS MADEIREIROS E NÃO MADEIREIROS}

A teca é uma das espécies florestais de melhor aceitação no mercado internacional de produtos madeireiros, sobre tudo pelas suas características de trabalhabilidade e durabilidade (MORA \& HERNANDÉZ, 2007). A densidade média da madeira é de, aproximadamente, $0,65 \mathrm{~g} \mathrm{~cm}^{-3} \mathrm{e}$, apesar da leveza, apresenta propriedades físico-mecânicas semelhantes as do mogno brasileiro, tais como durabilidade, estabilidade, facilidade de pré-tratamento, resistência natural ao ataque de fungos, insetos, pragas e brocas (CHAVES \& FONSECA, 1991; ABOD \& SIDDIQUI, 2002; VIEIRA et al., 2008; LUKMANDARU \& TAKAHASHI, 2009; GOMES et al., 2011).

Os maiores fabricantes de produtos industrializados a base de madeira de teca 
são a Indonésia, Tailândia, Índia e China (PANDEY \& BROWN, 2000), voltados a, aproximadamente, 25 usos distintos, desde a construção completa de uma casa até postes e peças de marcenaria (ALVARADO, 2006), entretanto, destaca-se à movelaria de luxo (Figura 4) e à construção naval (VRIEND, 1998; FIGUEIREDO et al., 2005b; NIAMKÉ et al., 2011), principalmente devido a elevada estabilidade dimensional, em associação com a estética visualmente agradável (MIRANDA et al., 2011).
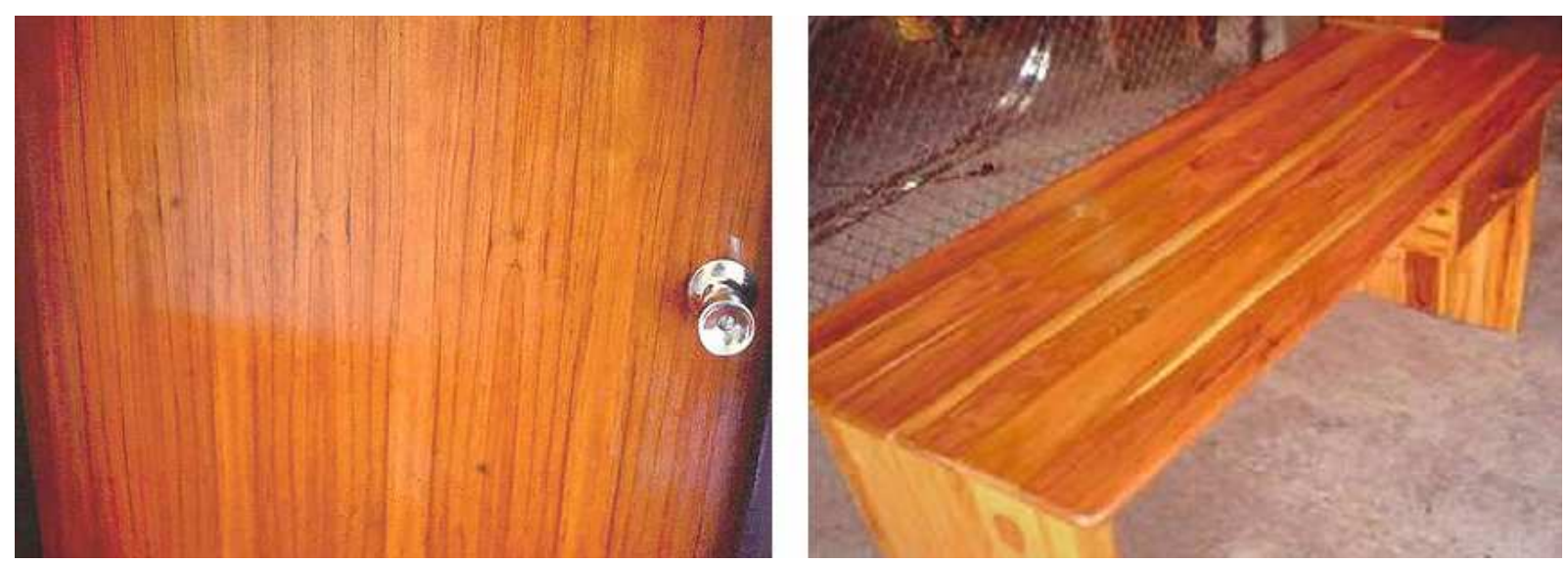

FIGURA 4 - Movelaria de madeira de teca. Fonte: LADRACH (2009).

Além disso, a madeira da espécie apresenta uma gama de finalidades, tais como a construção de bancos, cadeiras, pontes pequenas, teares, andaimes, produtos esculpido para decoração e utensílios domésticos, enquanto o aproveitamento de resíduos constitui uma alternativa para a produção de lenha (CATIE, 1986; VRIEND, 1998; MALDONADO \& LOUPPE, 1999).

As árvores de teca também são úteis como cercas vivas (CATIE, 1986; MALDONADO \& LOUPPE, 1999), enquanto as folhas são utilizadas como embalagens de açougue, extração de corantes para tingir seda e fármacos de combate à malária e anemia (CATIE, 1986; MALDONADO \& LOUPPE, 1999; GOMES et al., 2011), ao passo que, pesquisas recentes com extratos de flores, apontam o potencial antidiabético, antioxidante, anti-inflamatório, analgésico e de cicatrização de feridas (MAJUMDAR et al., 2007; BHATIA et al., 2011; RAMACHANDRAN et al., 2011a; RAMACHANDRAN et al., 2011b).

Atualmente, a madeira da teca é considerada uma alternativa às espécies de alto valor econômico, como a Swietenia macrophylla King e a Torresea acreana Ducke, para o suprimento sustentável das indústrias de base florestal (CALDEIRA et al., 2000; TSUKAMOTO FILHO et al., 2003; DRESCHER, 2004). Além da possibilidade de comercialização de produtos desde os primeiros desbastes (GONZÁLEZ, 2004; LADRACH, 2009), também existe o potencial para o mercado de fixação de carbono (ENTERS, 2000), o que torna a teca um investimento lucrativo aos seus produtores (ÂNGELO et al., 2009). 


\section{CARACTERÍSTICAS SILVICULTURAIS DOS PLANTIOS}

Nos trópicos, os plantios de teca têm alcançado maior auge nas últimas décadas devido ao potencial de crescimento e produtividade (VAIDES et al., 2005) e, atualmente, demonstra perspectiva alta de retorno de investimentos nos plantios intensivos (SHIMIZU et al., 2007). Entretanto, o cultivo da espécie é, por vezes, baseada em conhecimentos insuficientes (ENTERS, 2000), visto que a teca apresenta, em geral, crescimento superior nas Américas Central e do Sul, o que dificulta estabelecer comparações entre os regimes de manejo e as respostas das práticas de silvicultura em locais com características edafoclimáticas distintas (PÉREZ \& KANNINEN, 2005b; PELISSARI et al., 2013).

Normalmente, a densidade inicial dos plantios de teca é de 1.000 a 2.000 árvores.ha ${ }^{-1}$, com o primeiro desbaste entre quatro e cinco anos de idade e remoção de $50 \%$ da densidade inicial, para obter uma densidade final de 180 a 250 árvores.ha $^{-1}$ após uma rotação igual ou superior a 20 anos (FLOORS, 1997; PANDEY \& BROWN, 2000). Entretanto, na América Central, as densidades entre 1.110 a 1.600 árvores.ha ${ }^{-1}$, com três a cinco desbastes, são mais produtivos do que os espaçamentos mais amplos e com poucos desbastes (GONZÁLEZ, 2004).

No Brasil, geralmente os povoamentos de teca são implantados com 1.667 árvores.ha ${ }^{-1}$, espaçamento de $3,0 \mathrm{~m} \times 2,0 \mathrm{~m}$, e desbastes em torno de $5,10,15$ e 20 anos, o que proporcionam entre 200 a 250 árvores.ha $^{-1}$ para o corte final (GARCIA, 2006). No entanto, atualmente, há a tendência de aumento dos espaçamentos para 3,5 $\mathrm{m} \times 3,0 \mathrm{~m}$ ou $4,0 \mathrm{~m} \times 2,5 \mathrm{~m}$, principalmente com o advento de máquinas e implementos que exigem uma maior largura nas entrelinhas de plantio.

Nesses povoamentos implantados com objetivos comerciais, a aplicação de desbastes e desramas, desde os estágios iniciais de desenvolvimento, tem um efeito positivo sobre a forma do tronco da espécie, produzindo árvores com diâmetro e altura em proporções desejadas, fustes livres de nós, aumento do conteúdo de cerne e melhoria da qualidade sanitária dos plantios (CENTENO, 1997; KRISHNAPILLAY, 2000; SCHMINCKE, 2000; NOGUEIRA, 2003; PÉREZ, 2005; PÉREZ \& KANNINEN, 2005a; PELISSARI et al., 2013).

A teca é fortemente afetada pela competição intraespecífica (HÉRNANDEZ et al., 1993; CALDEIRA \& OLIVEIRA, 2008; PELISSARI et al., 2013), o que resulta na necessidade da execução de uma série de desbastes em diferentes intensidade e períodos. Dessa forma, a estratégia usual dos empreendimentos é manter os povoamentos com densidade inicial até o quarto ou quinto ano do plantio e, posteriormente, executar um desbaste seletivo com intensidades entre $40 \%$ a $60 \%$ do número de indivíduos por hectare (KRISHNAPILLAY, 2000; PANDEY \& BROWN, 2000; PÉREZ \& KANNINEN, 2003; KANNINEN et al., 2004; CRUZ, 2005; CALDEIRA \& OLIVEIRA, 2008).

As desramas, em geral, são executadas a partir do segundo ano, com a retirada de galhos até $1 / 3$ da altura total das árvores nessa idade, até a $1 / 2$ da altura total no terceiro ano e até $2 / 3$ no quarto ano, e a manutenção da desrama, com a remoção de galhos até 7,0 m de altura nas idades seguintes (SCHMINCKE, 2000; PÉREZ, 2005; PELISSARI, 2012).

A idade de rotação dos plantios de teca em sua área de distribuição natural varia entre 50 a 90 anos (PANDEY \& BROWN, 2000), com produtividade de 3 a 10 
$\mathrm{m}^{3} \cdot$ ha $^{-1} \cdot$ ano $^{-1}$ (CENTENO, 1997). Enquanto em outras regiões a rotação é mais curta, como no continente africano, com rotação de 35 a 55 anos e produtividade de 5 a 16 $\mathrm{m}^{3}$.ha ${ }^{-1}$.ano ${ }^{-1}$ (DUPUY \& VERHAEGEN, 1993; DUPUY et al., 1999); e nas Américas do Sul e Central, com expectativa de 20 a 25 anos (BERMEJO et al., 2004; BEZERRA, 2009; GONZÁLEZ, 2010) e produtividade de 10 a $20 \mathrm{~m}^{3}$.ha ${ }^{-1}$.ano ${ }^{-1}$ (CENTENO, 1997), com a possibilidade de valores maiores nos melhores sítios (MATA, 1999; DRESCHER, 2004; VAIDES et al., 2005).

\section{MODELAGENS APLICADAS AO MANEJO DA TECA}

A relação hipsométrica possibilita reduzir os custos dos inventários de plantios teca ao estimar a variável altura $(H)$ em função do diâmetro a $1,3 \mathrm{~m}$ do solo $(D A P)$. Ajustes estatisticamente precisos foram obtidos por DRESCHER (2004) com a equação $\sqrt{H}=1,390-0,063 \times D A P+0,448 \times \ln (D A P)^{2}$ em povoamentos em Mato Grosso, e por ROSSI et al. (2011) com $\frac{1}{H-1,3}=0,173+2,211 \times \frac{1}{D A P}-2,216 \times \ln \frac{1}{D A P^{2}}$ em plantios da espécie no Pará. Ao passo que, por meio de modelos com efeitos mistos, JAYARAMAN \& LAPPI (2001) verificaram predições adequadas da relação altura-diâmetro da teca em sítios específicos na Índia.

A possibilidade de determinar a variável volume $(V)$, por meio de modelos matemáticos, permite a predição das produções presente e futura dos povoamentos florestais. Estimativas precisas do volume em plantios de teca foram obtidas por PÉREZ \& KANINEN (2003) na Costa Rica, com as equações $\sqrt{V}=-0,088-0,029 \times D A P$ e $\ln (V)=0,000073 \times D A P^{1,559} \times H^{1,210}$, e por AKOSSOU et al. (2013) em Benin, África, com $\ln (V)=-1,990+1,73 \times \ln (D A P)+0,723 \times \ln (H)$. Além disso, MORET et al. (1998) na Venezuela e GARCÉS \& MORET (2001) e GÓMES \& MORA (2003) na Costa Rica determinaram diversas equações para estimativas de volumes total, comercial, com e sem casca para povoamentos de teca.

O índice de sítio, determinado pela altura dominante $\left(H_{\text {dom }}\right)$ em função do tempo ( $T$ ) em uma idade de referência, é uma ferramenta básica para a seleção de locais e de e para o manejo de plantios florestais (TORRES et al., 2012). No Brasil, CRUZ et al. (2008) determinaram que a equação $H_{d o m}=21,528 \times\left(1-e^{-0,025 \times T}\right)^{1,322}$ foi adequada para povoamentos de teca em Mato Grosso, ao passo que CONCEIÇÃO et al. (2012) concluíram que $\ln \left(H_{d o m}\right)=3,259-7,097 \times\left(\frac{1}{T}\right)+20,729 \times\left(\frac{1}{T}\right)^{2}-24,726 \times\left(\frac{1}{T}\right)^{3}$ foi apropriada para plantios no Pará. Ainda, SAJJADUZZAMAN et al. (2005) estabeleceram que a equação $H_{d o m}=27,960 \times\left(1-e^{-0,037 \times T}\right)^{1,081}$ foi satisfatória para a teca em Bangladesh, e TORRES et al. (2012) verificaram que o ajuste $H_{\text {dom }}=17,280 \times\left(1-e^{-1,960 \times T}\right)^{0,650}$ foi efetivo para a espécie na Colômbia.

Atualmente, as funções de afilamento são largamente utilizadas para estimar os diâmetros e os volumes ao longo dos fustes das árvores e para determinar os multiusos comerciais das seções dos troncos. Dessa forma, FAVALESSA et al. (2012a) e FAVALESSA et al. (2012b) verificaram que os modelos de Max \& Burkhart e de 
Hradetzky foram os mais acurados, respectivamente para as estimativas de volume e de diâmetro da base de fuste de teca em Mato Grosso, enquanto ADU-BREDU et al. (2008) descreveram a forma de árvores bifurcadas e LEITE et al. (2011) determinaram adequadamente o afilamento do cerne da espécie.

$\mathrm{Na}$ medida em que cresce a necessidade por informações detalhadas dos plantios florestais, consolida-se a utilização da geoestatística para a estimativa de uma variável em locais não amostrados e a aplicação em mapeamentos (GOMES et al., 2007; BORSSOI et al., 2011), assim como verificado por PELISSARI et al. (2012), que, por meio de modelagens espaciais, estabeleceram as correlações espaciais entre o desenvolvimento da teca e os atributos químicos do solo, o que possibilitou recomendar intervenções silviculturais direcionadas para as diferentes condições edáficas, visando a maximização da produção ao longo da rotação da cultura.

\section{CONSIDERAÇÕES FINAIS}

Atualmente, no mercado nacional e internacional, os plantios de teca são considerados como alternativas rentáveis e sustentáveis à exploração de espécies nativas, principalmente para a obtenção de madeira de uso nobre, tornando-os destaque na balança comercial do setor florestal brasileiro e uma oportunidade na conquista de mercados exigentes por produtos florestais de qualidade.

Para a ampliação da produção e da produtividade das florestas de teca, a seleção de matrizes genéticas de gerações de indivíduos adaptados as características edafoclimáticas do Brasil, e o posterior melhoramento genético, são atividades para viabilizar a implantação de futuros povoamentos altamente produtivos.

Além disso, a aplicação de técnicas de silvicultura de precisão, tal como a geoestatística, é uma alternativa para o zoneamento dos locais cultiváveis e a seleção de sítios com características propícias à implantação de florestas de teca, bem como a adequação da fertilidade natural dos solos às exigências fisiológicas da espécie e a mitigação de impactos ambientais negativos das práticas de cultivo.

Por fim, a otimização do manejo, por meio da definição ótima da intensidade e da frequência dos desbastes, da rotação técnica e econômica da cultura e do sortimento para múltiplos produtos, são possibilidades a serem trabalhadas para a minimização dos custos de condução dos povoamentos e para o incremento na rentabilidade dos produtores florestais.

\section{REFERÊNCIAS}

ABOD, S. A.; SIDDIQUI, M. T. Growth response of teak (Tectona grandis L.f.) seedlings to nitrogen, phosphorus and potassium fertilizers. Pertanika Journal of Tropical Agricultural Science, v. 25, n. 2, p. 107-113, 2002.

ABRAF - Associação Brasileira de Produtores de Florestas Plantadas. Anuário estatístico da ABRAF 2012, ano base 2011. Brasília: ABRAF, 2012. 150 p.

ADU-BREDU, S.; BI, A. F. T.; BOUILLET, J.; ME, M. K.; KYEI, S. Y.; SAINT-ANDRE, L. An explicit stem profile model for forked and un-forked teak (Tectona grandis) trees in West Africa. Forest Ecology and Management, n. 255, p. 2189-2203, 2008. 
AKOSSOU, A. Y. J.; ARZOUMA, S.; ATTAKPA, E. Y.; FONTON, N. H.; KOKOU, K. Scaling of teak (Tectona grandis) logs by the xylometer technique: accuracy of volume equations and influence of the log length. Diversity, v. 5, p. 99-113, 2013.

ALVARADO, A. Nutricion y fertilizacion de la teca. Informaciones Agronomicas, n. 61, p. 1-8, 2006.

ÂNGELO, H.; SILVA, V. S. de M.; SOUZA, Á. N. de; GATTO, A. C. Aspectos financeiros da produção de teca no Estado de Mato Grosso. Floresta, v. 39, n. 1, p. 23-32, 2009.

BEHLING, M. Nutrição, partição de biomassa e crescimento de povoamentos de teca em Tangará da Serra-MT. 2009. 176 f. Tese (Doutorado em Solos e Nutrição de Plantas) - Universidade Federal de Viçosa, Viçosa - MG.

BERMEJO, I.; CAÑELLAS, I.; SAN MIGUEL, A. Growth and yield models for teak plantations in Costa Rica. Forest Ecology and Management, v. 189, p. 97-110, 2004.

BEZERRA, A. F. Modelagem do crescimento e da produção de povoamentos de Tectona grandis submetidos a desbaste. 2009. 73 f. Dissertação (Mestrado em Ciência Florestal) - Universidade Federal de Viçosa, Viçosa - MG.

BHATIA, V.; SRIVASTAVA, S. P.; SRIVASTAVA, R.; MISHRA, A.; NARENDER, T.; MAURYA, R.; SRIVASTAVA, A. K. Antihyperglycaemic and aldose reductase inhibitory potential of Acacia catechu hard wood and Tectona grandis leaves. Medicinal Chemistry Research, v. 20, p. 1724-1731, 2011.

BORSSOI, J. A.; URIBE-OPAZO, M. A.; GALEA, M. Técnicas de diagnóstico de influência local na análise espacial da produtividade da soja. Engenharia Agrícola, v. 31, n. 2, p. 376-387, 2011.

CALDEIRA, B. R. P. P. Caracterização das propriedades físico-mecânicas e determinação da percentagem de cerne da madeira de Tectona grandis. Lisboa: Universidade Técnica de Lisboa, 2004. 84 p.

CALDEIRA, S. F.; CALDEIRA, S. A. F.; MENDONÇA, E. A. F. de; DINIZ, N. N. Caracterização e avaliação da qualidade dos frutos de teca (Tectona grandis L.f.) produzidos no Mato Grosso. Revista Brasileira de Sementes, v. 22, n. 1, p. 216-224, 2000.

CALDEIRA, S. F.; OLIVEIRA, D. L. C. Desbaste seletivo em povoamentos de Tectona grandis com diferentes idades. Acta Amazonica, v. 38, n. 2, p. 223-228, 2008.

CAMINO, R. de; MORALES, J. P. Las plantaciones de teca en América Latina: mitos y realidades. Turrialba: CATIE, 2013. 392 p. (Série técnica: Informe técnico, 397).

CASTELLANOS, A. F. R. Efecto del establecimiento de plantaciones forestales de 
Teca (Tectona grandis L.f.) en áreas de potrero sobre las características del suelo en Petén, Guatemala. 2006. 105 f. Dissertação (Mestrado em Agroforestería Tropical) - Centro Agronómico Tropical de Investigación y Enseñanza, Turrialba - Costa Rica.

CATIE - Centro Agronómico Tropical de Investigación y Enseñanza. Silvicultura de espécie promisorias para producción de leña en América Central: resultados de cinco años de investigación. Turrialba: CATIE, 1986. 228 p.

CENTENO, J. C. El manejo de las plantaciones de teca. Actualidad Forestal Tropical, v. 5 , n. 2, p. 10-12, 1997.

CHAVES, E.; FONSECA, W. Teca (Tectona grandis L.f.) árbol de uso múltiple em América Central. Turrialba: CATIE, 1991. 47 p. (Série técnica: Informe técnico, 179).

CRUZ, J. P. da. Crescimento e produção de Tectona grandis na região de Tangará da Serra - Mato Grosso. 2005. 57 f. Dissertação (Mestrado em Ciência Florestal) Universidade Federal de Viçosa, Viçosa - MG.

CRUZ, J. P.; LEITE, H. G., SOARES, C. P. B.; CAMPOS, J. C. C.; SMIT, L.; NOGUEIRA, G. S. Curvas de crescimento e de índice de local para povoamentos de Tectona grandis em Tangará da Serra, Mato Grosso. Revista Arvore, v. 32, n. 4, p. 679-685, 2008.

CONCEIÇÃO, F. X.; DRESCHER, R.; PELISSARI, A. L.; LANSSANOVA, L. R.; FAVALESSA, C. M. C.; ROQUETTE, J. G. Capacidade produtiva local de Tectona grandis em Monte Dourado, Estado do Pará, Brasil. Ciência Rural, v. 42, n. 5, p. 822827, 2012.

COSTA, R. B. da; RESENDE, M. D. V. de; SILVA, V. S. de M. e. Experimentação e seleção no melhoramento genético de TECA (Tectona grandis L.f.). Floresta e Ambiente, v. 14, n. 1, p. 76-92, 2007.

DRESCHER, R. Crescimento e produção de Tectona grandis Linn. F., em povoamentos jovens de duas regiões do Estado de Mato Grosso. 2004. $133 \mathrm{f}$. Tese (Doutorado em Engenharia Florestal) - Universidade Federal de Santa Maria, Santa Maria - RS.

DUPUY, B.; MAITTRE, H.; KANGA, A. N. Table de production du teck (Tectona grandis): L'exemple de la Côte d'Ivoire. Bois et Forêts des Tropiques, v. 3, n. 261, p. 5-16, 1999.

DUPUY, B.; VERHAEGEN, D. Le teck de plantation (Tectona grandis) en Côte d'Ivoire. Bois et Forêts des Tropiques, n. 235, p. 9-24, 1993.

ENTERS, T. Site, technology and productivity of teak plantations in Southeast Asia. Unasylva, v. 51, n. 201, p. 55-61, 2000. 
FAVALESSA, C. M. C.; UBIALLI, J. A.; CALDEIRA, S. F.; DRESCHER, R.; ACOSTA, F. C. Equações de sortimentos para Tectona grandis na região centro - sul de Mato Grosso. Pesquisa Florestal Brasileira, v. 32, n. 72, p. 389-399, 2012.

FAVALESSA, C. M. C.; UBIALLI, J. A.; CALDEIRA, S. F.; DRESCHER, R. Funções de afilamento não segmentadas e segmentadas para Tectona grandis na região centro-sul matogrossense. Pesquisa Florestal Brasileira, v. 32, n. 72, p. 373-387, 2012.

FIGUEIREDO, E. O.; OLIVEIRA, A. D. de; BARBOSA, L. K. F. Teca (Tectona grandis L.f.): principais perguntas do futuro empreendedor florestal. Rio Branco: Embrapa Acre, 2005. 87 p. (Embrapa Acre. Documentos, 97).

FIGUEIREDO, E. O.; OLIVEIRA, A. D. de; SCOLFORO, J. R. S. Análise econômica de povoamentos não desbastados de Tectona grandis L.f., na microrregião do baixo Rio Acre. Cerne, v. 11, n. 4, p. 342-353, 2005.

FLOORS, A. Plantation forestry in Guanacaste, Costa Rica: A lust description of plantation forestry (teak and melina) in Guanacaste, Costa Rica. Costa Rica: REPOSA, 1997. $49 \mathrm{p}$.

GARCÉS, A. M.; MORET, A. Y. Evalución de métodos de estimación para ajustar ecuaciones de volumen em plantaciones de teca (Tectona grandis L.). Revista Florestal Venezuelana, v. 45, n. 2, p. 63-67. 2001.

GARCIA, M. L. Intensidade de desbaste em um povoamento de Tectona grandis L.f., no município de Sinop - MT. 2006. 45 f. Dissertação (Mestrado em Agricultura Tropical) - Universidade Federal de Mato Grosso, Cuiabá - MT.

GOMES, I. M. da S.; SANTOS JUNIOR, W. R. dos; ARRUDA, A. da S. Análise de soluções para extração de corante de folhas da teca em diferentes estágios de desenvolvimento. Enciclopédia Biosfera, v. 7, n. 12, p. 1-18, 2011.

GOMES, N. M.; SILVA, A. M. da; MELLO, C. R. de; FARIA, M. A. de; OLIVEIRA, P. M. de. Métodos de ajuste e modelos de semivariograma aplicados ao estudo da variabilidade espacial de atributos físico-hídricos do solo. Revista Brasileira de Ciência do Solo, v. 31, p. 435-443, 2007.

GÓMEZ, Q, M.; MORA, F. A. Comparación de modelos y unificación de ecuaciones de volumen para árboles individuales en plantaciones de Teca (Tectona grandis Linn) en Costa Rica. Heredia: INISEFOR, 2003. 24 p.

GONZÁLEZ, S. A. S. Relación del suelo con el crecimiento inicial y contenido foliar de teca (Tectona grandis), y adaptación de leguminosas para control de arvenses bajo un sistema fertirriego en Campeche, México. 2010. 90 f. Dissertação (Mestrado em Agricultura Ecológica) - Centro Agronómico Tropical de Investigación y Enseñanza, 
Turrialba - Costa Rica.

GONZÁLEZ, W. F. Manual para productores de teca (Tectona grandis L. f) en Costa Rica. Heredia: Costa Rica, 2004. 121 p.

HERNÁNDEZ, R.; TORRES, A.; MÁRQUEZ, O.; FRANCO, W. Contenido foliar de nutrimentos y crecimiento en plantaciones de teca en Ticoporo, Ven. Turrialba, v. 43, n. 1, p. 11-15, 1993.

JAYARAMANA, K.; LAPPI, J. Estimation of height-diameter curves through multilevel models with special reference to even-aged teak stands. Forest Ecology and Management, v. 142, p. 155-162, 2001.

KANNINEN, M.; PÉREZ, D.; MONTEROB, M.; VÍQUEZ, E. Intensity and timing of the first thinning of Tectona grandis plantations in Costa Rica: results of a thinning trial. Forest Ecology and Management, n. 203, 89-99, 2004.

KAOSA-ARD, A. Overview of problems in teak plantation establishment. In: REGIONAL SEMINAR ON TEAK, 2., 1998, Yangon. Proceeding... Bangkok: FAO, 1998, p. 49-59.

KAOSA-ARD, A. Teak (Tectona grandis Linn. F.) natural distribution and related factors. Natural History Bulletin of the Siam Society, v. 29, p. 55-74, 1981.

KRISHNAPILLAY, B. Silviculture and management of teak plantations. Unasylva, v. 51, n. 201, p. 14-21, 2000.

LADRACH, W. Management of teak plantations for solid wood products. Bethesda: ISTF, 2009. 25 p.

LAMPRECHT, H. Silvicultura nos trópicos: ecossistemas florestais e respectivas espécies arbóreas. Rossdorf: TZ-Verl.-Ges., 1990. 343 p.

LEITE, H. G.; OLIVEIRA-NETO, R. R. de; MONTE, M. A.; FARDIN, L.; ALCANTARA, A. M. de; BINOTI, M. L. M. da S.; CASTRO, R. V. O. Modelo de afilamento de cerne de Tectona grandis L.f. Scientia Forestalis, v. 39, n. 89, p. 053-059, 2011.

LIMA, C. G. da R.; CARVALHO, M. de P. e; NARIMATSU, K. C. P.; SILVA, M. G. da; QUEIROZ, H. A. de. Atributos físico-químicos de um Latossolo do cerrado brasileiro e sua relação com características dendrométricas do eucalipto. Revista Brasileira de Ciência do Solo, v. 34, p. 163-173, 2010.

LIMA, I. L. de; FLORSHEIM, S. M. B.; LONGUI, E. L. Influência do espaçamento em algumas propriedades físicas da madeira de Tectona grandis Linn. Cerne, v. 15, n. 2, p. 244-250, 2009.

LUKMANDARU, G.; TAKAHASHI, K. Radial distribution of quinones in plantation teak 
(Tectona grandis L.f.). Annals of Forest Science, v. 66, p. 605-613, 2009.

MAJUMDAR, M.; NAYEEM, N.; KAMATH, J. V.; ASAD, M. Evaluation of Tectona grandis leaves for wound healing activity. Pakistan Journal of Pharmaceutical Sciences, v. 20, n. 2, p. 120-124, 2007.

MALDONADO, G.; LOUPPE, D. Les plantations villageoises de teck en Côte d'Ivoire. Bois et Forêts des Tropiques, n. 262, p. 9-30, 1999.

MATA, M. M. Factores de sitio que influyen en el crecimiento de Tectona grandis L. f. y Bombacopsis quinata (Jacq.) Dugand, en Costa Rica. 1999. 77 f. Dissertação (Mestrado em Ciências Florestais) - Universidad Austral de Chile, Valdivia - Chile.

MATRICARDI, W. A. T. Efeitos dos fatores de solo sobre o desenvolvimento da teca (Tectona grandis L. F.) cultivada na grande Cáceres - Mato Grosso. 1989. 135 f. Dissertação (Mestrado em Ciência Florestal) - Universidade de São Paulo, Piracicaba $-\mathrm{SP}$.

MIRANDA, I.; SOUSA, V.; PEREIRA, H. Wood properties of teak (Tectona grandis) from a mature unmanaged stand in East Timor. Journal of Wood Science, v. 57, p. 171$178,2011$.

MITTELMAN, A. Teak planting by smallholders in Nakhon Sawan, Thailand. Unasylva, v. 51, n. 201, p. 62-65, 2000.

MOLLINEDO GARCIA, M. S. Relación suelo-planta, factores de sitio y respuesta a la fertilización, en plantaciones jóvenes de teca (Tectona grandis L. f.), en la zona Oeste, Cuenca del canal de Panamá. 2003. 101 f. Dissertação (Mestrado em Agroforestería Tropical) - Centro Agronómico Tropical de Investigación y Enseñanza, Turrialba - Costa Rica.

MONTERO, M. M.; UGALDE, L.; KANNINEN, M. Relación del índice de sitio con los factores que influyen en el crescimento de Tectona grandis L. F. y Bombacopsis quinata (Jacq.) Dugand, en Costa Rica. Revista Forestal Centroamericana, n. 35, p. 13-18, 2001.

MORA, F.; HERNÁNDEZ, W. Estimación del volumen comercial por producto para rodales de teca en el pacífico de Costa Rica. Agronomía Costarricense, v. 31, n. 1, p. 101-112, 2007.

MORAES, L. F. D. de; CAMPELlO, E. F. de C.; PEREIRA, M. G.; LOSS, A. Características do solo na restauração de áreas degradadas na Reserva Biológica de Poço das Antas, RJ. Ciência Florestal, v. 18, n. 2, p. 193-206, 2008.

MORET, A. Y.; JEREZ, M.; MORA, A. Determinación de ecuaciones de volumen para plantaciones de teca (Tectona grandis L.) en la unidad experimental de la Reserva 
Forestal Caparo, Estado Barinas - Venezuela. Revista Florestal Venezuelana, v. 42, n. 1, p. 41-50, 1998.

NAIR, C. T. S.; SOUVANNAVONG, O. Emerging research issues in the management of teak. Unasylva, v. 51, n. 201, p. 45-54, 2000.

NEWBY, J. C.; CRAMB, R. A.; SAKANPHET, S.; McNAMARA, S. Small holder teak and agrarian change in Northern Laos. Small-scale Forestry, v. 11, p. 27-46, 2012.

NIAMKÉ, F. B.; AMUSANT, N.; CHARPENTIER, J.; CHAIX, G.; BAISSAC, Y.; BOUTAHAR, N.; ADIMA, A. A.; KATI-COULIBALY, S.; JAY-ALLEMAND, C. Relationships between biochemical attributes (non-structural carbohydrates and phenolics) and natural durability against fungi in dry teak wood (Tectona grandis L. f.). Annals of Forest Science, v. 68, p. 201-211, 2011.

NOCETTI, M.; ROZENBERG, P.; CHAIX, G.; MACCHIONI, N. Provenance effect on the ring structure of teak (Tectona grandis L.f.) wood by X-ray microdensitometry. Annals of Forest Science, v. 68, p. 1375-1383, 2011.

NOGUEIRA, G. S. Modelagem do crescimento e da produção de povoamentos de Eucalyptus sp. e de Tectona grandis submetidos a desbaste. 2003. 145 f. Tese (Doutorado em Ciência Florestal) - Universidade Federal de Viçosa, Viçosa - MG.

OLIVEIRA, J. R. V. de. Sistema para cálculo de balanço nutricional e recomendação de calagem e adubação de povoamentos de teca - Nutriteca. 2003. 89 f. Dissertação (Mestrado em Solos e Nutrição de Plantas) - Universidade Federal de Viçosa, Viçosa - MG.

OMBINA, C. A. Soil characterization for teak (Tectona grandis) plantations in Nzara District of South Sudan. 2008. 135 f. Dissertação (Mestrado em Ciências Florestais) Université des Sciences et Techniques de Masuku - Gabão.

PANDEY, D.; BROWN, C. Teak: a global overview. Unasylva, v. 51, n. 201, p. 3-13, 2000.

PELISSARI, A. L. Silvicultura de precisão aplicada ao desenvolvimento de Tectona grandis L.f. na região Sul do estado de Mato Grosso. 2012. 78 f. Dissertação (Mestrado em Ciências Florestais e Ambientais) - Universidade Federal de Mato Grosso, Cuiabá - MT.

PELISSARI, A. L.; CALDEIRA, S. F.; DRESCHER, R. Desenvolvimento Quantitativo e Qualitativo de Tectona grandis L.f. em Mato Grosso. Floresta e Ambiente, v. 20, n. 3, p. 371-383, 2013.

PELISSARI, A. L.; CALDEIRA, S. F.; SANTOS, V. S. dos; SANTOS, J. O. P. dos. Correlação espacial dos atributos químicos do solo com o desenvolvimento da teca em 
Mato Grosso. Pesquisa Florestal Brasileira, v. 32, n. 71, p. 247-256, 2012.

PÉREZ, D. Stand growth scenarios for Tectona grandis plantations in Costa Rica. 2005. 77 f. Dissertação (Mestrado em Agricultura e Silvicultura) - Universidade de Helsinque, Helsinque, Finlândia.

PÉREZ, D.; KANNINEN, M. Effect of thinning on stem form and wood characteristics of teak (Tectona grandis) in a humid tropical site in Costa Rica. Silva Fennica, v. 39, n. 2, p. 217-225, 2005.

PÉREZ, D.; KANNINEN, M. Provisional equations for estimating total and merchantable volume of Tectona grandis trees in Costa Rica. Forests, Trees and Livelihoods, v. 13, p. 345-359, 2003.

PÉREZ, D.; KANNINEN, M. Stand growth scenarios for Tectona grandis plantations in Costa Rica. Forest Ecology and Management, n. 210, p. 25-441, 2005.

RAMACHANDRAN, S.; RAJASEKARAN, A.; KUMAR, K. M. Antidiabetic, antihyperlipidemic and antioxidant potential of methanol extract of Tectona grandis flowers in streptozotocin induced diabetic rats. Asian Pacific Journal of Tropical Medicine, p. 624-631, 2011.

RAMACHANDRAN, S.; RAJINI, K. B.; RAJASEKARAN, A.; KUMAR, K. M. Evaluation of anti-inflammatory and analgesic potential of methanol extract of Tectona grandis flowers. Asian Pacific Journal of Tropical Biomedicine, p. 155-158, 2011.

ROSSI, A. S.; DRESCHER, R.; PELISSARI, A. L.; LANSSANOVA, L. R. Relação hipsométrica e crescimento de Tectona grandis L.f. no município de Monte Dourado, Pará. Scientia Forestalis, v. 39, n. 91, p. 301-307, 2011.

RUGMINI, P.; JAYARAMAN, K. Intrinsic units of growth for teak trees. Trees, v. 23, p. 51-58. 2009.

SAJJADUZZAMAN, M.; MOLLICK, A.; MITLOHNER, R.; MUHAMMED, N.; KAMAL, M. Site index for teak (Tectona grandis Linn. F.) in forest plantations of Bangladesh. International Journal of Agriculture and Biology, v. 7, n. 4, p. 547-549, 2005.

SALAZAR, R.; ALBERTIN, W. Requerimentos edaficos y climáticos para Tectona grandis. Turrialba, v. 1, n. 24, p. 66-71, 1974.

SCHMINCKE, K. H. Teak plantations in Costa Rica: Precious Woods' experience. Unasylva, v. 51, n. 201, p. 29-35, 2000.

SCHUHLI, G. S.; PALUDZYSZYN FILHO, E. O cenário da silvicultura de teca e perspectivas para o melhoramento genético. Pesquisa Florestal Brasileira, v. 30, n. 63, p. 217-230, 2010. 
SHIMIZU, J. Y.; KLEIN, H.; OLIVEIRA, J. R. V. Diagnóstico das plantações florestais em Mato Grosso. Cuiabá: Central de Texto, 2007. 63 p.

SHUKLA, P. K.; RAO, R. V.; SHASHIKALA, S.; KUMAR, P.; SHARMA, S. K. Wood quality variation in Tectona grandis (teak) clones from CSO raised at Maredumilli (Rajahmundry), Andhra Pradesh. Journal of the Indian Academy of Wood Science, v. 8, n. 2, p. 116-119, 2011.

SILVA, A. M. da; MORAES, M. L. T. de; BUZETTI, S. Propriedades químicas de solo sob reflorestamento ciliar após 20 anos de plantio em área de cerrado. Revista Árvore, v. 35, n. 1, p. 97-106, 2011.

SINHA, S. K.; DEEPAK, M. S.; RAO, R. V.; BORGAONKAR, H. P. Dendroclimatic analysis of teak (Tectona grandis L. f.) annual rings from two locations of peninsular India. Current Science, v. 100, n. 1, p. 84-88, 2011.

SUZUKI, R.; TAKEDA, S.; THEIN, H. M. Chronosequence changes in soil properties of teak (Tectona grandis) plantations in the Bago Mountains, Myanmar. Journal of Tropical Forest Science, v. 19, n. 4, p. 207-217, 2007.

TANAKA, N.; HAMAZAKI, T.; VACHARANGKURA, T. Distribution, growth and site requirements of teak. Japan Agricultural Research Quarterly, v. 32, p. 65-77, 1998.

TECA DO BRASIL. Galerias / A Floresta. 2013. Disponível em: $<\mathrm{http}: / / w w w . t e c a d o b r a s i l . c o m . b r / p t / g a l e r i a s / g a l e r i a . a s p ? i d=1>$. Acesso em: 24 jan. 2014.

TONINI, H.; COSTA, M. C. G. C.; SCHWENGBER, L. A. M. Crescimento da teca (Tectona grandis) em reflorestamento na Amazônia Setentrional. Pesquisa Florestal Brasileira, n. 59, p. 05-14, 2009.

TORRES, D. A.; VALLE, J. I.; RESTREPO, G. Site index for teak in Colombia. Journal of Forestry Research, v. 23, n. 3, p. 405-411, 2012.

TROPICOS. Missouri botanical garden. 2013. Disponível em: <http://www.tropicos.org>. Acesso em: 08 nov. 2013.

TSUKAMOTO FILHO, A. de A.; SILVA, M. L. da; COUTO, L.; MÜLLER, M. D. Análise econômica de um plantio de teca submetido a desbastes. Revista Árvore, v. 27, n. 4, p. 487-494, 2003.

UPADHYAY, A.; EID, T.; SANKHAYAN, P. L. Construction of site index equations for even aged stands of Tectona grandis (teak) from permanent plot data in India. Forest Ecology and Management, n. 212, p. 14-22, 2005. 
VAIDES LÓPEZ, E. E. Características de sitio que determinan el crecimiento y productividad de teca (Tectona grandis L.f.), en plantaciones forestales de diferentes regiones en Guatemala. 2004. 81 f. Dissertação (Mestrado em Ciencias sobre Manejo y Conservación de Bosques Tropicales y Biodiversidad) - Centro Agronómico Tropical de Investigación y Enseñanza, Turrialba - Costa Rica.

VAIDES, E.; UGALDE, L.; GALLOWAY, G. Crecimiento y productividad de teca en plantaciones forestales jóvenes en Guatemala. Recursos Naturales y Ambiente, n. 46, p. 137-145, 2005.

VALLEJOS BARRA, O. S. Productividad y relaciones del índice de sitio con variables fisiográficas, edafoclimáticas y foliares para Tectona grandis L.F., Bombacopsis quinatum (Jacq.) Dugand y Gmelina arborea Roxb. en Costa Rica. 1996. 168 f. Dissertação (Mestrado em Ciencias Agrícolas y Recursos Naturales) Centro Agronómico Tropical de Investigación y Enseñanza, Turrialba - Costa Rica.

VÁSQUEZ, W. C.; UGALDE, L. A. A. Rendimiento y calidad de sitio para Gmelina arborea, Tectona grandis, Bombacopsis quinatum y Pinus caribaea en Guanacaste, Costa Rica. Turrialba: CATIE, 1995. 40 p. (Serie técnica: Informe técnico, n. 256).

VIEIRA, A. H.; ROCHA, R. B.; BENTES-GAMA, M. de M.; LOCATELLI, M. Desempenho de teca (Tectona grandis) em plantio adensado no Estado de Rondônia. Porto Velho: Embrapa Rondônia, 2008. 14 p. (Boletim de Pesquisa e Desenvolvimento n. 56).

VRIEND, J. de. Teak: an exploration of market prospects and the outlook for Costa Rican plantations based on indicative growth tables. Turrialba: CATIE, 1998. 77 p.

ZECH, W.; DRECHSEL, P. Relationships between growth, mineral nutrition and site factors of teak (Tectona grandis) plantations in the rainforest zone of Liberia. Forest Ecology and Management, v. 41, n. 3-4, p. 221-235. 1991. 